\title{
SINGULARITIES IN DERIVED CATEGORIES
}

\author{
BERNT TORE JENSEN AND XIUPING SU
}

\begin{abstract}
Let $A$ be a finite dimensional algebra over an algebraically closed field $k$ and let $M$ and $N$ be two complexes in the bounded derived category $D^{b}(A)$ of finitely generated $A$ modules. Together with Alexander Zimmermann we have defined a notion of degeneration for derived categories. We say that $M$ degenerates to $N$ if there is a complex $Z$ and an exact triangle $N \longrightarrow M \oplus Z \longrightarrow Z \longrightarrow N[1]$. In this paper we define study the type of singularity at every degeneration in the bounded derived categrory.
\end{abstract}

\section{INTRODUCTION AND MAIN RESULTS}

Let $A$ be a finite dimensional algebra over an algebraically closed field $k$. Let $a_{1}, \ldots, a_{s}$ be generators of $A$. For any positive integer $d$ let $\bmod (A, d)$ denote the variety of $d$-dimensional $A$-modules. So a point in $\bmod (A, d)$ is an $s$-tuple of $d$ by $d$ matrices satisfying the same relations as the generators $a_{1}, \ldots, a_{s}$. The group $G l_{d}$ acts on $\bmod (A, d)$ by conjugation and the orbits correspond to isomorphism classes of $d$-dimensional $A$-modules. We write $M \leq_{\text {deg }} N$ if $N \in \overline{G l_{d} \cdot M}$. In this case one says that $M$ degenerates to $N$.

Following Hesselink [7] we say that two pointed varieties $\left(X, x_{0}\right)$ and $\left(Y, y_{0}\right)$ are smoothly equivalent if there is a pointed variety $\left(Z, z_{0}\right)$ and a pair of smooth morphisms

$$
\left(X, x_{0}\right) \stackrel{f}{\longleftarrow}\left(Z, z_{0}\right) \stackrel{g}{\longrightarrow}\left(Y, y_{0}\right)
$$

with $f\left(z_{0}\right)=x_{0}$ and $g\left(z_{0}\right)=y_{0}$. This is an equivalence relation on pointed varieties. Following Zwara [14] we denote the equivalence class of $\left(X, x_{0}\right)$ by $\operatorname{Sing}\left(X, x_{0}\right)$. The class $\operatorname{Sing}\left(X, x_{0}\right)$ is called the type of singularity of $X$ at $x_{0}$. Two pointed varieties with the same type of singularity share many geometric properties, like for example being normal or Cohen-Macaulay. For modules $M$ and $N$ with $M \leq_{d e g} N$ we write $\operatorname{Sing}(M, N)$ for the type of singularity $\operatorname{Sing}\left(\overline{G l_{d} \cdot M}, N\right)$.

Several mathematicians have studied the types of singularities which occur when taking orbit closures in module varieties. For example Bongartz [4] has shown that type of singularity is preserved under Morita equivalence. He also classified singularities for minimal degenerations in module varieties of hereditary algebras of Dynkin type and showed that type of singularity is preserved under classical tilting [5]. Zwara has studied what happens with type of singularities under exact functors [14]. He used his results to show, together with Bobinski, that orbit closures for hereditary algebras of type $\mathbb{A}[2]$ and $\mathbb{D}[3]$ are among other things normal and Cohen-Macaulay. Skowronski and Zwara proved that type of singularity is preserved under derived equivalence of self-injective algebras [13].

The main aim of this note is to show that investigations of type of singularity is also possible for degenerations in the bounded derived category. We show that there is a natural notion of type of singularity at every degeneration in the bounded derived category of finitely generated $A$-modules, which is well-defined up to the equivalence relation given by Hesselink. We show that the type of singularitiy of a degeneration of modules coincide with the type of singularity in the corresponding degeneration of projective resolutions. For hereditary algebras we also study what happens with the type of singularity of a degeneration of bounded complexes when passing to homology.

We recall a notion of varieties for derived categories [9] which was defined in collaboration with Alexander Zimmermann. Similar varieties have been studied by Huisgen-Zimmermann

Date: preliminary version of April 24, 2005. 
and Saorín [8] and Bekkert and Drozd [1]. Let $P_{1}, \ldots, P_{l}$ be a complete set of representatives of projective indecomposable $A$-modules. For every sequence $\underline{d}: \mathbb{Z} \longrightarrow \mathbb{N}^{l}$ for which there is an $i_{0} \in \mathbb{Z}$ with $d_{i}=(0, \ldots, 0)$ for $i \leq i_{0}$ we define $\operatorname{comproj}(A, \underline{d})$ to be the subset of

$$
\prod_{i \in \mathbb{Z}} \operatorname{Hom}_{A}\left(\bigoplus_{j=1}^{l} P_{j}^{d_{i}^{j}}, \bigoplus_{j=1}^{l} P_{j}^{d_{i-1}^{j}}\right)
$$

consisting of sequences of maps $\left(\partial_{i}\right)_{i \in \mathbb{Z}}$ such that $\partial_{i-1} \partial_{i}=0$ for all $i \in \mathbb{Z}$. Such a sequence $\underline{d}$ is called a dimension array.

The group

$$
G=G_{\underline{d}}:=\prod_{i \in \mathbb{Z}} A u t_{A}\left(\bigoplus_{j=1}^{l} P_{j}^{d_{i}^{j}}\right)
$$

acts on comproj $(A, \underline{d})$ by conjugation and two complexes in $\operatorname{comproj}(A, \underline{d})$ are in the same orbit if and only if they are quasi-isomorphic ([9], Lemma 2).

We say that a dimension array $\underline{d}$ is bounded if there is an $i_{1} \in \mathbb{Z}$ with $d_{i}=(0, \ldots, 0)$ for $|i| \geq i_{1}$. In this case comproj $(A, \underline{d})$ is an affine variety and in particular it has the Zariski topology and moreover $G$ is a connected algebraic group.

For any dimension array $\underline{d}$ we let $\underline{d}_{n}$ be the dimension array with $\left(d_{n}\right)_{i}=d_{i}$ for $i \leq n$ and $\left(d_{n}\right)_{i}=0$ otherwise. Naive truncation on the left at degree $n$ induces surjective maps

$$
\pi_{n}: \operatorname{comproj}(A, \underline{d}) \longrightarrow \operatorname{comproj}\left(A, \underline{d}_{n}\right)
$$

where $\pi_{n}\left(\left(\partial_{i}\right)_{i \in \mathbb{Z}}\right)_{j}=\partial_{j}$ for $j \leq n$.

We give comproj $(A, \underline{d})$ the weak topology with respect to the maps $\left\{\pi_{n}\right\}_{n \in \mathbb{Z}}$. That is, $\operatorname{comproj}(A, \underline{d})$ is the projective limit of the varieties $\operatorname{comproj}\left(A, \underline{d}_{n}\right)$ in the category of topological spaces. Let $\varphi_{n}$ be the truncation map from $\operatorname{comproj}\left(A, \underline{d}_{n}\right)$ to $\operatorname{comproj}\left(A, \underline{d}_{n-1}\right)$ for all $n \in \mathbb{Z}$. Note that the action of $G$ on $\operatorname{comproj}(A, \underline{d})$ induces an action of $G$ on $\operatorname{comproj}\left(A, \underline{d}_{n}\right)$, for all $n$, making $\pi_{n}$ and $\varphi_{n} G$-equivariant.

Let $N$ and $M$ be complexes in the bounded derived category of finitely generated $A$ modules $D^{b}(A)$. We say that $M$ degenerates to $N$ if there is a complex $Z$ and an exact triangle

$$
N \longrightarrow M \oplus Z \longrightarrow Z \longrightarrow N[1]
$$

In this case we write $M \leq_{\operatorname{deg}} N$. From [9] we know that for any two complexes $M$ and $N$ there exists a dimension array $\underline{d}$ and complexes $M^{\prime}, N^{\prime} \in \operatorname{comproj}(A, \underline{d})$ such that $M$ and $N$ are quasi-isomorphic to $M^{\prime}$ and $N^{\prime}$, respectively. The main result of our collaboration with Alexander Zimmermann [9] is that for any such choice of $M^{\prime}$ and $N^{\prime}$ we have $M \leq_{\operatorname{deg}} N$ if and only if $N^{\prime} \in \overline{G \cdot M^{\prime}}$ in comproj $(A, \underline{d})$. Note that it is possible for two complexes to be quasi-isomorphic without having the same dimension array. This flexibility is needed in order to get a good theory of degeneration. So our joint work with Alexander Zimmermann raises a natural question, which geometric properties of an orbit closure are invariant under quasi-isomorphism? Our main result of this paper provides an answer.

Let $M, N \in D^{b}(A)$ with $M \leq_{\text {deg }} N$ and assume that the homology of $N$ vanishes in degrees greater than or equal to $t$. We define

$$
\operatorname{Sing}(M, N):=\operatorname{Sing}\left(\overline{G \cdot \pi_{t+1}\left(M^{\prime}\right)}, \pi_{t+1}\left(N^{\prime}\right)\right),
$$

where $M^{\prime}$ and $N^{\prime}$ are right bounded complexes of projective $A$-modules such that $M \simeq M^{\prime}$ and $N \simeq N^{\prime}$ and where $N^{\prime}$ and $M^{\prime}$ have the same dimension array. Clearly this definition depends on the choice of $M^{\prime}, N^{\prime}$ and $t$. Our main result says that all choices gives the same type of singularity.

Theorem 1. The type of singularity $\operatorname{Sing}(M, N)$ is well defined.

From [9] we know that the degeneration relation for modules is compatible with the degeneration relation in the derived category. 
Proposition 1. [9] Let $M, N \in \bmod (A, d)$ for some dimension $d$ and let $P_{M}, P_{N} \in$ $\operatorname{comproj}(A, \underline{d})$ for some dimension array $\underline{d}$ be projective resolutions of $M$ and $N$, respectively. Then $M \leq_{\text {deg }} N$ in $\bmod (A, d)$ if and only if $P_{M} \leq_{\text {deg }} P_{N}$ in $\operatorname{comproj}(A, \underline{d})$.

Our second main result compares the singularities in an orbit closure of a module with the singularities in the orbit closure of the corresponding projective resolution.

Theorem 2. Let $M, N \in \bmod (A, d)$ for some dimension $d$ and let $P_{M}, P_{N} \in \operatorname{comproj}(A, \underline{d})$ for some dimension array $\underline{d}$ be projective resolutions of $M$ and $N$, respectively. If $M \leq_{\text {deg }} N$ then $\operatorname{Sing}(M, N)=\operatorname{Sing}\left(P_{M}, P_{N}\right)$.

We may view $A$ as a $\mathbb{Z}$-graded algebra, concentrated in degree 0 . Let $X=\bigoplus_{i \in \mathbb{Z}} X_{i}$ and $Y=\bigoplus_{i \in \mathbb{Z}} Y_{i}$ be finite dimensional $\mathbb{Z}$-graded A-modules. We say that $X$ degenerate to $Y$ if $X_{i}$ degenerate to $Y_{i}$ for each $i \in \mathbb{Z}$. In this case we write $X \leq_{\operatorname{deg}} Y$. If $X$ degenerates to $Y$ we let $\operatorname{Sing}(X, Y)$ denote the type of singularity

$$
\operatorname{Sing}(X, Y):=\operatorname{Sing}\left(\prod_{i \in \mathbb{Z}} \overline{G l_{n_{i}} \cdot X_{i}},\left(Y_{i}\right)_{i \in \mathbb{Z}}\right)
$$

where $n_{i}=\operatorname{dim}_{k} X_{i}$. Note that to each complex $M$ we can associate its $\mathbb{Z}$-graded homology module $H_{*}(M)=\bigoplus_{i \in \mathbb{Z}} H_{i}(M)$. We say that two complexes $M$ and $N$ have homology of equal dimension if $\operatorname{dim}_{k} H_{i}(M)=\operatorname{dim}_{k} H_{i}(N)$ for each $i \in \mathbb{Z}$. It is known that, for hereditary algebras, complexes are determined up to quasi-isomorphism by their homology. We show that taking homology preserves the types of singularities.

Theorem 3. Let $A$ be a hereditary algebra. Let $M, N \in D^{b}(A)$ with homology of equal dimension. Then $M \leq_{\text {deg }} N$ if and only if $H_{*}(M) \leq_{\text {deg }} H_{*}(N)$ and in this case $\operatorname{Sing}(M, N)=$ $\operatorname{Sing}\left(H_{*}(M), H_{*}(N)\right)$.

By the results of Bobinski and Zwara [2] [3] we get the following immeadiate corollary.

Corollary 2. Let $A$ be a hereditary algebra of type $\mathbb{A}$ or $\mathbb{D}$. Let $M, N \in \operatorname{comproj}(A, \underline{d})$ be bounded complexes with homology of equal dimension. If $M \leq_{\operatorname{deg}} N$ then the singularity of $N$ in the orbit closure of $M$ is normal and Cohen-Macaulay.

The remainder of this paper is organized as follows. In Section 1 we show that type of singularity is preserved when truncating complexes. In Section 2 we prove that type of singularity is preserved by adding contractible complexes. As a consequence, Theorem 1 follows. Finally, Theorem 2 and Theorem 3 are proven in Section 3 and Section 4, respectively.

\section{Type OF SINGUlarity is PRESERVED UNDER TRUNCATION}

For two bounded complexes $M$ and $N$ with the same dimension array such that $M \leq_{\operatorname{deg}} N$, let

$$
\operatorname{Sing}(M, N):=\operatorname{Sing}(\overline{G \cdot M}, N) .
$$

The main result of this section is the following

Proposition 3. Let $\underline{d}$ be any dimension array and let $M, N \in \operatorname{comproj}(A, \underline{d})$ be two complexes with bounded homology such that $M \leq_{\operatorname{deg}} N$. If the homology of $N$ vanishes in degrees greater than or equal to $t$ then $\operatorname{Sing}\left(\pi_{m}(M), \pi_{m}(N)\right)=\operatorname{Sing}\left(\pi_{m+1}(M), \pi_{m+1}(N)\right)$ for all $m>t$.

Before we prove the proposition we need some preparation. Recall that a function $f$ : $X \longrightarrow \mathbb{Z}$ on a topological space $X$ is called upper (resp. lower) semicontinous if for each $n \in \mathbb{Z}$ the subspace $\{x \in X \mid f(x) \leq n\}$ is open (resp. closed) in $X$.

Lemma 4. Let $n$ be an integer. The function $h_{n}: \operatorname{comproj}(A, \underline{d}) \longrightarrow \mathbb{Z}$ given by $h_{n}(X)=$ $\operatorname{dim}_{k} H_{n}(X)$ is upper semicontinous. 
Proof. Without loss of generality we may assume that $\underline{d}$ is bounded. The subset $\{X \in$ $\left.\operatorname{comproj}(A, \underline{d}) \mid \operatorname{dim}_{k} i m \partial_{n}^{X} \geq l\right\}$ of $\operatorname{comproj}(A, \underline{d})$ is given by the non-vanishing of a minor and is therefore open. This shows that the functions $X \mapsto \operatorname{dim}_{k} k e r \partial_{n}^{X}$ and $X \mapsto$ $\operatorname{dim}_{k} i m \partial_{n+1}^{X}$ is upper and lower semicontinous, respectively. Thus $h_{n}(X)=\operatorname{dim}_{k} k e r \partial_{n}^{X}-$ $\operatorname{dim}_{k} i m \partial_{n+1}^{X}$ is upper semicontinous.

We will use the following basic lemma on singularities, without reference, throughout the remainder of this paper.

Lemma 5. Let $(X, x)$ be a pointed variety.

i) If $U \subseteq X$ is an open neighborhood of $x$ then $\operatorname{Sing}(U, x)=\operatorname{Sing}(X, x)$.

ii) If $X$ is smooth at $x$ then $\operatorname{Sing}(X \times Y,(x, y))=\operatorname{Sing}(Y, y)$ for any $(Y, y)$.

Proof. It is well known that an open immersion is a smooth morphism, hence $i$ ) follows. Now $i$ ) follows from the fact that the projection $X \times Y \longrightarrow Y$ is smooth if $X$ is smooth.

We recall a well-known result on vector bundles.

Proposition 6 (See [10][12]). Let $f: E \longrightarrow F$ be a map of vector bundles over $X$. Suppose that the rank of $f_{x}$ remains constant as $x$ varies over $X$. Then ker $f$ and imf are sub-bundles of $E$ and $F$, respectively.

Let $\mathfrak{r}$ denote the Jacobson radical of $A$. Note that since $k$ is algebraically closed there is an injective algebra homomorphism $A / \mathfrak{r} \longrightarrow A$ such that the composition $A / \mathfrak{r} \longrightarrow A \longrightarrow A / \mathfrak{r}$ is the identity. We let any $A$-module be an $A / \mathfrak{r}$-module via the inclusion $A / \mathfrak{r} \longrightarrow A$.

Lemma 7. Let $\underline{d}$ be any dimension array and let $m$ be an integer. Let $U \subseteq \operatorname{comproj}\left(A, \underline{d}_{m}\right)$ be a locally closed subset with the property that the isomorphism class of ker $\partial_{m}^{X}$ as an $A / \mathfrak{r}$ module is constant for all $X \in U$. Then $\varphi_{m+1}^{-1}(U) \longrightarrow U$ is a vector bundle.

Proof. Let $M_{i}=\bigoplus_{j=1}^{l} P_{j}^{d_{i}^{j}}$ for all $i \in \mathbb{Z}$. For each $X \in U$ the fibre $\varphi_{m+1}^{-1}(X)$ is isomorphic to $\operatorname{Hom}_{A}\left(M_{m+1}, \operatorname{ker}_{m}^{X}\right)$. By assumption $\operatorname{ker} \partial_{m}^{X}$ is constant as an $A / \mathfrak{r}$-module for all $X \in U$. Hence the dimension of the fibre is constant since $M_{m+1}$ is a projective $A$-module.

Now $\varphi_{m+1}^{-1}(U) \longrightarrow U$ is the kernel of the map of trivial vector bundles $\operatorname{Hom}_{A}\left(M_{m+1}, M_{m}\right) \times$ $U \longrightarrow \operatorname{Hom}_{A}\left(M_{m+1}, M_{m-1}\right) \times U$ given by sending a pair $\left(f,\left(\partial_{i}^{X}\right)_{i}\right)$ to $\left(\partial_{m}^{X} \circ f,\left(\partial_{i}^{X}\right)_{i}\right)$. Thus the lemma follows from Proposition 6.

Proof of Proposition 3. Let $M, N \in \operatorname{comproj}(A, \underline{d})$ with $M \leq_{d e g} N$. Assume that $H_{n}(N)=$ 0 for all $n \geq t$ and let $m>t$ be an integer. Note that by Lemma $4 H_{n}(M)=0$ whenever $H_{n}(N)=0$. Let $U_{m} \subseteq \overline{G \cdot \pi_{m}(M)}$ consist of the complexes $X$ with $H_{n}(X)=0$ for all $n=t, \ldots, m-1$. Then $\pi_{m}(M), \pi_{m}(N) \in U_{m}$ and moreover $U_{m}$ is open in $\overline{G \cdot \pi_{m}(M)}$ by Lemma 4. Thus $\operatorname{Sing}\left(U_{m}, \pi_{m}(N)\right)=\operatorname{Sing}\left(\pi_{m}(M), \pi_{m}(N)\right)$.

We show that for any $X \in U_{m}$ we have $k e r \partial_{m}^{X} \simeq k e r \partial_{m}^{M}$ as $A / \mathfrak{r}$-modules. For if $X \in U_{m}$ then $\pi_{m}(M) \leq_{\text {deg }} X$ and so there exist a complex $Z$ and an exact triangle $X \longrightarrow \pi_{m}(M) \oplus$ $Z \longrightarrow Z \longrightarrow X[1]$. By taking homology we get an exact sequence

$$
0 \longrightarrow \operatorname{ker}_{m}^{X} \longrightarrow \operatorname{ker}_{m}^{M} \oplus H_{m}(Z) \longrightarrow H_{m}(Z) \longrightarrow H_{m-1}(X) \text {. }
$$

But $H_{m-1}(X)=0$ and so $k e r \partial_{m}^{X} \simeq k e r \partial_{m}^{M}$ as $A / \mathfrak{r}$-modules.

By Lemma 7 the map $\varphi_{m+1}^{-1}\left(U_{m}\right) \longrightarrow U_{m}$ is a vector bundle and so $\operatorname{Sing}\left(U_{m}, \pi_{m}(N)\right)=$ $\operatorname{Sing}\left(\varphi_{m+1}^{-1}\left(U_{m}\right), \pi_{m+1}(N)\right)$, since $\varphi_{m+1}\left(\pi_{m+1}(N)\right)=\pi_{m}(N)$.

Let $U_{m+1} \subseteq \varphi_{m+1}^{-1}\left(U_{m}\right)$ denote the subset of complexes $X$ with $H_{m}(X)=0$. Then $U_{m+1}$ is open in $\varphi_{m+1}^{-1}\left(U_{m}\right)$ by Lemma 4 . By assumption we have $\pi_{m+1}(M), \pi_{m+1}(N) \in U_{m+1}$. Now $G \cdot \pi_{m+1}(M) \subseteq U_{m+1}$ and $G \cdot \pi_{m+1}(M) \subseteq \varphi_{m+1}^{-1}\left(G \cdot \pi_{m}(M)\right)$. On the other hand if $Y \in \varphi_{m+1}^{-1}\left(G \cdot \pi_{m}(M)\right)$ then $\varphi_{m}(Y) \simeq \varphi_{m}(M)$. Moreover if in addition $H_{m}(Y)=0$ then $Y \in G \cdot \pi_{m+1}(M)$. This shows that

$$
G \cdot \pi_{m+1}(M)=U_{m+1} \cap \varphi_{m+1}^{-1}\left(G \cdot \pi_{m}(M)\right)
$$


which is open in $\varphi_{m+1}^{-1}\left(U_{m}\right)$. By Lemma 7 we know that $\varphi_{m+1}^{-1}\left(U_{m}\right)$ is irreducible and so $\operatorname{Sing}\left(\varphi_{m+1}^{-1}\left(U_{m}\right), \pi_{m+1}(N)\right)=\operatorname{Sing}\left(\pi_{m+1}(M), \pi_{m+1}(N)\right)$. This completes the proof of the proposition.

\section{Type of SINGULARITY IS PRESERVED When ADDING CONTRACTIBLE COMPLEXES}

Theorem 1 follows from Proposition 3 and the following

Proposition 8. Let $f$ and $f^{\prime}$ be bounded dimension arrays. Let $M, N \in \operatorname{comproj}(A, f)$ and $M^{\prime}, N^{\prime} \in \operatorname{comproj}\left(A, f^{\prime}\right)$ be complexes such that $M \leq_{\text {deg }} N$. If $M \simeq M^{\prime}$ and $N \simeq \overline{N^{\prime}}$ then $\operatorname{Sing}(M, N)=\operatorname{Sing}\left(\bar{M}^{\prime}, N^{\prime}\right)$.

We need some preparation before proving the proposition. We will prove several lemmas involving short exact sequences of complexes. Analogous results for short exact sequences of modules can be found in [11] and [6].

Let $E$ be a bounded contractible complex of projective $A$-modules. We denote the abelian category of bounded complexes of finitely generated $A$-modules by $C^{b}(A)$. Let $\underline{d}$ be the dimension array of $E$ and let $\underline{e}$ and $f$ be dimension arrays with $\underline{d}=\underline{e}+f$. Here addition of dimension arrays is done componentwise.

Lemma 9. Let $\underline{e}, \underline{f}$ and $E$ be as above.

i) $\operatorname{dim}_{k} \operatorname{Hom}_{C^{b}(A)}(E, Y)$ is constant for all $Y \in \operatorname{comproj}(A, f)$.

ii) $\operatorname{dim}_{k} \operatorname{Hom}_{C^{b}(A)}(X, E)$ is constant for all $X \in \operatorname{comproj}(A, \underline{\underline{e}})$.

Proof. We may assume that $E$ has the form

$$
E: \ldots \longrightarrow 0 \longrightarrow P \stackrel{1}{\longrightarrow} P \longrightarrow 0 \longrightarrow \ldots
$$

for a projective $A$-module $P$. This is sufficient because any bounded contractible complex of projective $A$-modules is a direct sum of complexes of this form. Let $E$ be concentrated in degrees $i$ and $i+1$. Let $Y$ be a complex in $\operatorname{comproj}(A, f)$. Then $H_{O^{b}(A)}(E, Y) \simeq$ $\operatorname{Hom}_{A}\left(P, Y_{i+1}\right)$, which has constant dimension for all $Y$ in $\operatorname{comproj}(A, f)$.

Similarly for a complex $X$ in $\operatorname{comproj}(A, \underline{e})$ we have $\operatorname{Hom}_{C^{b}(A)}\left(X, \overline{E)} \simeq \operatorname{Hom}_{A}\left(X_{i}, P\right)\right.$, which has constant dimension as well. This proves $i i$ ).

For a subset $U \subseteq \operatorname{comproj}(A, \underline{e})$ let $\operatorname{Fac}(U, E)$ denote the subset of $\operatorname{comproj}(A, \underline{f})$ consisting of complexes $Y$ for which there exists a short exact sequence of complexes

$$
0 \longrightarrow X \longrightarrow E \longrightarrow Y \longrightarrow 0
$$

with $X$ in $U$. Dually for a subset $V$ of $\operatorname{comproj}(A, f)$ let $S u b(E, V)$ be the subset of $\operatorname{comproj}(A, \underline{e})$ consisting of complexes $X$ for which there exists a short exact sequence $0 \longrightarrow X \longrightarrow E \longrightarrow Y \longrightarrow 0$ with $Y$ in $V$. Let $F a c(E, f)=F a c(\operatorname{comproj}(A, \underline{e}), E)$ and let $\operatorname{Sub}(\underline{e}, E)=\operatorname{Sub}(E, \operatorname{comproj}(A, \underline{f}))$. Moreover let $\operatorname{Sub}(E, M)=S u b(E,\{M\})$ for $M$ in $\operatorname{comproj}(A, f)$ and let $\operatorname{Fac}\left(M^{\circ}, E\right)=F a c\left(\left\{M^{\circ}\right\}, E\right)$ for $M^{\circ}$ in $\operatorname{comproj}(A, \underline{e})$.

We view $\bar{A}$ as a graded algebra concentrated in degree 0 . Any complex becomes a graded $A$-module by forgetting the differential. For two graded $A$-modules $X$ and $Y$ we denote by $\operatorname{Hom}_{A}(X, Y)_{0}$ the graded homomorphisms of degree zero. Let $Q$ and $R$ be the graded $A$-modules with

$$
Q_{i}=\bigoplus_{j=1}^{l} P_{j} e_{i}^{j} \text { and } R_{i}=\bigoplus_{j=1}^{l} P_{j} f_{i}^{j},
$$

respectively. Then any complex in $\operatorname{comproj}(A, \underline{e})$ is equal to $Q$ as a graded $A$-module. Similarly any complex in $\operatorname{comproj}(A, f)$ is equal to $R$ as a graded $A$-module.

Let $T(\underline{e}, \underline{f})$ be the locally closed subset of $\operatorname{Hom}_{A}(Q, E)_{0} \times \operatorname{Hom}_{A}(E, R)_{0}$ consisting of pairs $(\phi, \theta)$ such that

$$
0 \longrightarrow Q \stackrel{\phi}{\longrightarrow} E \stackrel{\theta}{\longrightarrow} R \longrightarrow 0
$$


is a short exact sequence of graded $A$-modules with the image of $\phi$ a subcomplex of $E$. Let $\operatorname{Surj}(E, f)$ be the locally closed subset of $\operatorname{Hom}_{A}(E, R)_{0}$ consisting of surjective homomorphisms $\theta: E \longrightarrow R$ such that the kernel of $\theta$ is a subcomplex of $E$. Note that any map $\theta$ in $\operatorname{Surj}(E, f)$ is a split graded homomorphism.

Lemma 10. The split injective homomorphisms in $\operatorname{Hom}_{A}(Q, E)_{0}$ form an open subset.

Proof. Any graded $A$-module becomes a graded $A / \mathfrak{r}$-module via the inclusion $A / \mathfrak{r} \longrightarrow A$ of Section 1. Let $V$ be a graded $A / \mathfrak{r}$-complement to $\mathfrak{r} Q$ in $Q$ and let $W$ be a graded $A / \mathfrak{r}$-complement to $\mathfrak{r} E$ in $E$. Then any homomorphism $f \in \operatorname{Hom}_{A}(Q, E)_{0}$ has the form

$$
f=\left(\begin{array}{ll}
\alpha & \beta \\
\gamma & \delta
\end{array}\right): V \oplus \mathfrak{r} Q \longrightarrow W \oplus \mathfrak{r} E
$$

We have a map $\operatorname{Hom}_{A}(Q, E)_{0} \longrightarrow \operatorname{Hom}_{A / \mathfrak{r}}(V, W)_{0}$ given by sending $f$ to $\alpha$. Then the subset of split injective homomorphisms in $\operatorname{Hom}_{A}(Q, E)$ is the inverse image of the open subset of injective homomorphisms in $\operatorname{Hom}_{A / \mathfrak{r}}(V, W)_{0}$, and therefore an open subset.

Let $\operatorname{Inj}(\underline{e}, E)$ be the locally closed subset of $\operatorname{Hom}_{A}(Q, E)_{0}$ consisting of split injective graded homomorphisms $\phi: Q \longrightarrow E$ such that the image of $\phi$ is a subcomplex of $E$. Let $\rho_{1}: T(\underline{e}, \underline{f}) \longrightarrow \operatorname{Inj}(\underline{e}, E)$ and $\rho_{2}: T(\underline{e}, \underline{f}) \longrightarrow \operatorname{Surj}(E, \underline{f})$ be the natural projection maps. The group $G_{\underline{e}} \times G_{f}$ acts on $T(\underline{e}, \underline{f})$ by $\left(\bar{g}_{1}, g_{2}\right)(\phi, \theta)=\left(\bar{\phi} \circ\left(g_{1}\right)^{-1}, g_{2} \circ \theta\right)$. Similarly, $G_{\underline{e}}$ acts on $\operatorname{Inj}(\underline{e}, E)$ and $\bar{G}_{\underline{f}}$ acts on $\operatorname{Surj}(E, \underline{f})$.

Lemma 11. Let $\rho_{1}$ and $\rho_{2}$ be as above.

i) The map $\rho_{1}$ is a $G_{\underline{e}}$-equivariant principal $G_{\underline{f}}$-bundle locally trivial in the Zariski topology.

ii) The map $\rho_{2}$ is a $G_{\underline{f}}$-equivariant principal $G_{\underline{\underline{e}}}$-bundle locally trivial in the Zariski topology.

Proof. It suffices to prove part $i)$. Part $i i)$ is dual. The fibre of a map $\phi \in \operatorname{Inj}(\underline{e}, E)$ is given by all surjective maps $\theta: E \longrightarrow R$ with kernel the image of $\phi$. Thus the fibre is isomorphic to the degree zero graded automorphisms of $R$, which is $G_{\underline{f}}$. What remains is to show local triviality. Let $\phi$ be a map in $\operatorname{Inj}(\underline{e}, E)$. Then $\phi$ is split by definition. Let $V$ be a graded submodule complement to the image of $\phi$ in $E$. Let $I_{\phi}$ be the subset of $\operatorname{Inj}(\underline{e}, E)$ consisting of maps $\phi^{\prime}$ such that $i m \phi^{\prime} \cap V=0$. Then $I_{\phi}$ is a nonempty open subset of $\operatorname{Inj}(\underline{e}, E)$. For any $\left(\phi^{\prime}, \theta\right) \in \rho_{1}^{-1}\left(I_{\phi}\right)$ we have

$$
\phi^{\prime}=\left(\begin{array}{l}
\alpha \\
\beta
\end{array}\right): Q \longrightarrow i m \phi \oplus V \text { and } \theta=\left(\begin{array}{ll}
\gamma & \delta
\end{array}\right): i m \phi \oplus V \longrightarrow R
$$

with $\alpha$ an isomorphism. Now $\gamma \alpha+\delta \beta=0$ and so $\gamma=-\delta \beta \alpha^{-1}$. Hence $\rho_{1}^{-1}\left(I_{\phi}\right) \longrightarrow I_{\phi}$ is isomorphic to the trivial bundle $I_{\phi} \times G_{f} \longrightarrow I_{\phi}$ where $\left(\phi^{\prime}, \theta\right)$ is mapped to $\left(\phi^{\prime}, \delta\right)$. By varying the choice of $\phi$ we get a covering of $\operatorname{Inj}(\underline{e}, E)$, which shows local triviality.

Let $\gamma_{2}: \operatorname{Surj}(E, \underline{f}) \longrightarrow F a c(E, \underline{f})$ be the morphism of varieties which sends a graded homomorphism $\theta: \bar{E} \longrightarrow R$ to the unique point $Y$ in $\operatorname{comproj}(A, f)$ which makes $\theta: E \longrightarrow$ $Y$ a homomorphism of complexes. That $Y$ is unique is clear. We have to show that $\gamma_{2}$ is a morphism of varieties. Let $\theta \in \operatorname{Sur} j(E, f)$. Let $V$ be a graded $A$-submodule complement to the kernel of $\theta$ in $E$. Let $I_{\theta}$ be the affine open subset of all morphisms $\gamma$ in $\operatorname{Surj}(E, f)$ such that $\gamma$ restricted to $V$ is an isomorphism of graded modules. That is, $\gamma$ has the form

$$
\gamma=(\alpha, \beta): \operatorname{ker} \theta \oplus V \longrightarrow R
$$

where $\beta$ is a graded isomorphism. Now $\gamma_{2}$ restricted to $I_{\theta}$ is given by

$$
\gamma_{2}(\gamma)=\left(\begin{array}{ll}
\alpha & \beta
\end{array}\right) \partial^{E}\left(\begin{array}{c}
0 \\
\beta^{-1}
\end{array}\right)
$$


and is therefore a morphism of varieties. By varying the choice of $\theta$ we get a covering of $\operatorname{Surj}(E, f)$ by open subsets, showing that $\gamma_{2}$ is a morphism of varieties.

Similarly let $\gamma_{1}: \operatorname{Inj}(\underline{e}, E) \longrightarrow S u b(\underline{e}, E)$ be the morphism sending a graded homomorphism $\phi: Q \longrightarrow E$ to the unique point $X$ in $S u b(\underline{e}, E)$ which makes $\phi: X \longrightarrow E$ a homomorphism of complexes.

Lemma 12. Let $\gamma_{1}$ and $\gamma_{2}$ be as above.

i) There is a vector bundle $\delta_{1}: V_{1} \longrightarrow \operatorname{comproj}(A, \underline{e})$ such that $\operatorname{Inj}(\underline{e}, E)$ can be identified with an open subset of $V_{1}$ in such a way that $\gamma_{1}$ is the restriction of $\delta_{1}$ to $\operatorname{Inj}(\underline{e}, E)$. In particular $\operatorname{Sub}(\underline{e}, E)$ is open in comproj $(A, \underline{e})$.

ii) There is a vector bundle $\delta_{2}: V_{2} \longrightarrow \operatorname{comproj}(A, f)$ such that $\operatorname{Surj}(E, f)$ can be identified with an open subset of $V_{2}$ in such a way that $\gamma_{2}$ is the restriction of $\delta_{2}$ to $\operatorname{Surj}(E, \underline{f})$. In particular $\operatorname{Fac}(E, \underline{f})$ is open in $\operatorname{comproj}(A, \underline{f})$.

Proof. It suffices to prove part $i$ ). Part ii) is dual. Let $V_{1} \subseteq \operatorname{comproj}(A, \underline{e}) \times \operatorname{Hom}_{A}(Q, E)_{0}$ be the closed subset of pairs $(X, \phi)$ such that $\phi: X \longrightarrow E$ is an homomorphism of complexes. Let $\delta_{1}: V_{1} \longrightarrow \operatorname{comproj}(A, \underline{e})$ be the restriction of the trivial vector bundle $\operatorname{comproj}(A, \underline{e}) \times \operatorname{Hom}_{A}(Q, E)_{0} \longrightarrow \operatorname{comproj}(A, \underline{e})$ to $V_{1}$. Then $\delta_{1}^{-1}(X)=\operatorname{Hom}_{C^{b}(A)}(X, E)$ which has constant dimension by Lemma 9. Now $\delta_{1}: V_{1} \longrightarrow \operatorname{comproj}(A, \underline{e})$ is a vector bundle by Proposition 6 , since it is the kernel of the morphism of trivial vector bundles $\operatorname{comproj}(A, \underline{e}) \times \operatorname{Hom}_{A}(Q, E)_{0} \longrightarrow \operatorname{comproj}(A, \underline{e}) \times \operatorname{Hom}_{A}(Q, E[1])_{0}$ where $(X, f)$ is mapped to $\left(X, \partial^{X} f-f \partial^{X}\right)$. The map $\epsilon: \operatorname{Inj}(\underline{e}, E) \longrightarrow V_{1}$ given by $\epsilon(\phi)=\left(\gamma_{1}(\phi), \phi\right)$ is an isomorphism onto the subset of pairs $(X, \phi)$ with $\phi$ split injective as a homomorphism of graded modules. This is an open subset by Lemma 10. Thus $\operatorname{Sub}(\underline{e}, E)$ is open in $\operatorname{comproj}(A, \underline{e})$, since vector bundles are open morphisms. This completes the proof of part $i$ ).

Lemma 13. Let

$$
\begin{gathered}
0 \longrightarrow X \longrightarrow E \longrightarrow Y \longrightarrow 0 \\
0 \longrightarrow X^{\prime} \longrightarrow E \longrightarrow Y^{\prime} \longrightarrow 0
\end{gathered}
$$

be short exact sequences with $X, X^{\prime} \in S u b(\underline{e}, E)$ and $Y, Y^{\prime} \in F a c(E, f)$. Then $X^{\prime} \simeq X$ if and only if $Y^{\prime} \simeq Y$.

Proof. From the short exact sequences we get triangles $X \longrightarrow 0 \longrightarrow Y \longrightarrow X[1]$ and $X^{\prime} \longrightarrow 0 \longrightarrow Y^{\prime} \longrightarrow X^{\prime}[1]$. The result follows from the five-lemma for triangles.

We are now ready to prove Proposition 8.

Proof of Proposition 8. Let $f$ be a bounded dimension array and let $M, N \in \operatorname{comproj}(A, f)$ be complexes with $M \leq_{\operatorname{deg}} \bar{N}$. It suffices to show that $\operatorname{Sing}(M, N)=\operatorname{Sing}(M \oplus C, N \oplus \bar{C})$ for any contractible complex $C$. This is because by Lemma 2 in [9] there exists contractible complexes $C$ and $C^{\prime}$ such that $M \oplus C$ and $M^{\prime} \oplus C^{\prime}$ have the same dimension array. We may assume that $C$ is bounded by the definition of $\operatorname{Sing}(M, N)$ and Proposition 3.

Let $E$ be a bounded contractible complex of projective $A$-modules such that there are surjective homomorphisms $E \longrightarrow M$ and $E \longrightarrow N$ in the abelian category of bounded complexes. Let $\underline{d}$ be the dimension array of $E$ and let $\underline{e}=\underline{d}-\underline{f}$.

For any $U \subseteq S u b(\underline{e}, E)$ the corresponding subset $\operatorname{Fac}(U, E) \subseteq F a c(E, \underline{f})$ is given by

$$
\operatorname{Fac}(U, E)=\gamma_{2} \rho_{2} \rho_{1}^{-1} \gamma_{1}^{-1}(U)
$$

Similarly $\operatorname{Sub}(E, V)=\gamma_{1} \rho_{1} \rho_{2}^{-1} \gamma_{2}^{-1}(V)$ for any subset $V \subseteq$ Fac $(E, f)$.

Now let $M^{\circ} \in \operatorname{Sub}(E, M)$ and $N^{\circ} \in S u b(E, N)$. By Lemma 11 and Lemma 12 we get

$$
\operatorname{Sing}(M, N)=\operatorname{Sing}\left(\left(\gamma_{2} \rho_{2}\right)^{-1}\left(\overline{G_{\underline{f}} \cdot M} \cap \operatorname{Fac}(E, \underline{f})\right), \tilde{N}\right) .
$$

for any $\tilde{N}$ with $\gamma_{2} \rho_{2}(\tilde{N}) \simeq N$. Dually, we have

$$
\operatorname{Sing}\left(M^{\circ}, N^{\circ}\right)=\operatorname{Sing}\left(\left(\gamma_{1} \rho_{1}\right)^{-1}\left(\overline{G_{\underline{e}} \cdot M^{\circ}} \cap \operatorname{Sub}(\underline{e}, E)\right), \tilde{N}^{\circ}\right) .
$$


for any $\tilde{N}^{\circ}$ with $\gamma_{1} \rho_{1}\left(\tilde{N}^{\circ}\right) \simeq N^{\circ}$. Moreover by Lemma 13 , we have $\gamma_{2} \rho_{2}\left(\tilde{N}^{\circ}\right) \simeq N$ and $\gamma_{1} \rho_{1}(\tilde{N}) \simeq N^{\circ}$.

We have

$$
\overline{\gamma_{1}^{-1}\left(G_{\underline{e}} \cdot M^{\circ}\right)}=\gamma_{1}^{-1}\left(\overline{G_{\underline{e}} \cdot M^{\circ}} \cap S u b(\underline{e}, E)\right)
$$

For clearly $\overline{\gamma_{1}^{-1}\left(G_{\underline{e}} \cdot M^{\circ}\right)} \subseteq \gamma_{1}^{-1}\left(\overline{G_{\underline{e}} \cdot M^{\circ}} \cap \operatorname{Sub}(\underline{e}, E)\right)$. Now by Lemma $12 \gamma_{1}^{-1}\left(\overline{G_{\underline{e}} \cdot M^{\circ}} \cap\right.$ $\operatorname{Sub}(\underline{e}, E))$ is an irreducible closed subset of $\operatorname{Inj}(\underline{e}, E)$. Hence the equality follows.

A similar argument using Lemma 11 shows that

$$
\left(\gamma_{1} \rho_{1}\right)^{-1}\left(\overline{G_{\underline{e}} \cdot M^{\circ}} \cap \operatorname{Sub}(\underline{e}, E)\right)=\overline{\left(\gamma_{1} \rho_{1}\right)^{-1}\left(G_{\underline{e}} \cdot M^{\circ}\right)} .
$$

Also

$$
\left(\gamma_{2} \rho_{2}\right)^{-1}\left(\overline{G_{\underline{f}} \cdot M} \cap \operatorname{Fac}(E, \underline{f})\right)=\overline{\left(\gamma_{2} \rho_{2}\right)^{-1}\left(G_{\underline{f}} \cdot M\right)} .
$$

Now by Lemma 13 we have $\left(\gamma_{2} \rho_{2}\right)^{-1}\left(G_{\underline{f}} \cdot M\right)=\left(\gamma_{1} \rho_{1}\right)^{-1}\left(G_{\underline{e}} \cdot M^{\circ}\right)$. Hence

$$
\left(\gamma_{1} \rho_{1}\right)^{-1}\left(\overline{G_{\underline{e}} \cdot M^{\circ}} \cap \operatorname{Sub}(\underline{e}, E)\right)=\left(\gamma_{2} \rho_{2}\right)^{-1}\left(\overline{G_{\underline{f}} \cdot M} \cap \operatorname{Fac}(E, \underline{f})\right)
$$

Consequently $\operatorname{Sing}(M, N)=\operatorname{Sing}\left(M^{\circ}, N^{\circ}\right)$.

Now let $E^{\prime}=E \oplus C$. Then dually to the above $\operatorname{Sing}\left(M^{\circ}, N^{\circ}\right)=\operatorname{Sing}\left(M^{\prime \prime}, N^{\prime \prime}\right)$ where $M^{\prime \prime} \in \operatorname{Fac}\left(E^{\prime}, M^{\circ}\right)$ and $N^{\prime \prime} \in \operatorname{Fac}\left(E^{\prime}, N^{\circ}\right)$. But $M^{\prime \prime} \simeq M \oplus C$ and $N^{\prime \prime} \simeq N \oplus C$, again by Lemma 13. Thus $\operatorname{Sing}(M, N)=\operatorname{Sing}(M \oplus C, N \oplus C)$. This completes the proof of the proposition.

\section{Proof of Theorem 2}

As in the statement of the theorem let $M, N \in \bmod (A, d)$ for some dimension $d$ and let $P_{M}, P_{N} \in \operatorname{comproj}(A, \underline{d})$ for some dimension array $\underline{d}$ be projective resolutions of $M$ and $N$, respectively. Assume that $M \leq_{d e g} N$. By Proposition 3 we have $\operatorname{Sing}\left(P_{M}, P_{N}\right)=$ $\operatorname{Sing}\left(\pi_{2}\left(P_{M}\right), \pi_{2}\left(P_{N}\right)\right)$. Let $U \subseteq \overline{G \cdot \pi_{1}\left(P_{M}\right)}$ be the open subset of complexes $X$ with $H_{0}(X) \cong H_{0}\left(P_{M}\right)$ as $A / \mathfrak{r}$-modules. Then $\varphi_{2}^{-1}(U) \longrightarrow U$ is a vector bundle by Lemma 7 . Thus $\operatorname{Sing}\left(\pi_{1}\left(P_{M}\right), \pi_{1}\left(P_{N}\right)\right)=\operatorname{Sing}\left(\varphi_{2}^{-1}(U), \pi_{2}\left(P_{N}\right)\right)$.

Let $U^{\prime} \subseteq \varphi_{2}^{-1}(U)$ denote the subset of complexes $X$ with $H_{1}(X)=0$. Then $U^{\prime}$ is open in $\varphi_{2}^{-1}(U)$ by Lemma 4 . Now $Y \in \varphi_{2}^{-1}\left(G \cdot \pi_{1}\left(P_{M}\right)\right) \cap U^{\prime}$ if and only if $Y$ is the first three terms of a projective resolution of $M$. This shows that

$$
G \cdot \pi_{2}\left(P_{M}\right)=U^{\prime} \cap \varphi_{2}^{-1}\left(G \cdot \pi_{1}\left(P_{M}\right)\right)
$$

which is open in $\varphi_{2}^{-1}(U)$.

Now $\varphi_{2}^{-1}(U)$ is irreducible and so $\operatorname{Sing}\left(\pi_{2}\left(P_{M}\right), \pi_{2}\left(P_{N}\right)\right)=\operatorname{Sing}\left(\varphi_{2}^{-1}(U), \pi_{2}\left(P_{N}\right)\right)$. The theorem now follows from the following main lemma.

Lemma 14. $\operatorname{Sing}\left(\pi_{1}\left(P_{M}\right), \pi_{1}\left(P_{N}\right)\right)=\operatorname{Sing}(M, N)$.

We need some preparation before proving the lemma. Some of our constructions are similar to [11]. We denote the module at degree $i$ of the complex $P_{M}$ by $Q_{i}$. Let $\operatorname{Surj}_{A}\left(Q_{0}, d\right)$ denote the variety of surjective $k$-homomorphisms $\mu: Q_{0} \longrightarrow k^{d}$ with the property that the kernel of $\mu$ is an $A$-submodule of $Q_{0}$. Let $\pi: \operatorname{Surj}_{A}\left(Q_{0}, d\right) \longrightarrow \bmod (A, d)$ be the morphism given by sending $\mu$ to the unique $A$-module which makes $\mu$ an $A$-homomorphism. Let $\bmod (A, \mathbf{d})$ be the $G l_{d^{-}}$closed subset of $\bmod (A, d)$ consisting of all the modules which are isomorphic to $M$ as an $A / \mathfrak{r}$-module. By [4] we know that $\bmod (A, \mathbf{d})$ is a connected component of $\bmod (A, d)$. Let $\operatorname{Surj}_{A}\left(Q_{0}, \mathbf{d}\right)=\pi^{-1}(\bmod (A, \mathbf{d}))$ and let $F a c(Q, \mathbf{d})=\pi\left(\operatorname{Surj}_{A}\left(Q_{0}, \mathbf{d}\right)\right)$.

Lemma 15. Let $V_{1}=\left\{(Y, f) \in \bmod (A, \mathbf{d}) \times \operatorname{Hom}_{k}\left(Q_{0}, \mathrm{k}^{d}\right) \mid f: Q_{0} \longrightarrow Y\right.$ is an A-homomorphism $\}$. Let $\phi_{1}: \bmod (A, \mathbf{d}) \times \operatorname{Hom}_{k}\left(Q_{0}, \mathrm{k}^{d}\right) \longrightarrow \bmod (A, \mathbf{d})$ be the trivial vector bundle. Then the restriction $\left.\phi_{1}\right|_{V_{1}}: V_{1} \longrightarrow \bmod (A, \mathbf{d})$ is a vector bundle. 
Proof. Let $Y_{i}$ and $Q_{i}^{\prime}$ for $i=1, \ldots, s$ denote the matrices determined by the action of the $i$-th generator $a_{i}$ of $A$ on $Y$ and $Q_{0}$, respectively. Let

$$
\phi_{2}: \bmod (A, \mathbf{d}) \times \prod_{i=1}^{s} \operatorname{Hom}_{k}\left(Q_{0}, \mathrm{k}^{d}\right) \longrightarrow \bmod (A, \mathbf{d})
$$

be the trivial vector bundle. Now $V_{1}$ is the kernel of the morphism of the two trivial vector bundles $\phi_{1}$ and $\phi_{2}$, defined by sending $(Y, f)$ to $\left(Y,\left(Y_{i} f-f Q_{i}^{\prime}\right)_{i}\right)$. Moreover the kernel on the fibre of $Y$ is $\operatorname{Hom}_{A}\left(Q_{0}, Y\right)$ which has constant dimension as $Y$ varies over $\bmod (A, \mathbf{d})$, since $Q_{0}$ is projective and the isomorphism class of $Y$ as an $A / \mathfrak{r}$-module is constant. Hence by Proposition 6 the restriction of $\phi_{1}$ to $V_{1}$ is a vector bundle.

Let $\phi_{3}: \operatorname{Hom}_{A}\left(Q_{1}, Q_{0}\right) \times \operatorname{Surj}_{A}\left(Q_{0}, \mathbf{d}\right) \longrightarrow \operatorname{Hom}_{A}\left(Q_{1}, Q_{0}\right)$ and $\phi_{4}: \operatorname{Hom}_{A}\left(Q_{1}, Q_{0}\right) \times$ $\operatorname{Surj}_{A}\left(Q_{0}, \mathbf{d}\right) \longrightarrow \operatorname{Surj}_{A}\left(Q_{0}, \mathbf{d}\right)$ be the two natural projections. Note that $\operatorname{Hom}_{A}\left(Q_{1}, Q_{0}\right)=$ $\operatorname{comproj}\left(A, \underline{d}_{1}\right)$.

Lemma 16. Let $V_{2}=\left\{(\lambda, \mu) \in \operatorname{Hom}_{A}\left(Q_{1}, Q_{0}\right) \times \operatorname{Surj}_{A}\left(Q_{0}, \mathbf{d}\right) \mid \mu \lambda=0\right\}$. Then the restriction $\left.\phi_{4}\right|_{V_{2}}: V_{2} \longrightarrow \operatorname{Surj}_{A}\left(Q_{0}, \mathbf{d}\right)$ is a vector bundle.

Proof. Let $\phi_{5}: \operatorname{Hom}_{\mathrm{k}}\left(Q_{1}, \mathrm{k}^{d}\right) \times \operatorname{Surj}_{A}\left(Q_{0}, \mathbf{d}\right) \longrightarrow \operatorname{Surj}_{A}\left(Q_{0}, \mathbf{d}\right)$ be the trivial vector bundle. Now $V_{2}$ is the kernel of the morphism between the two trivial vector bundles $\phi_{4}$ and $\phi_{5}$, given by sending $(\lambda, \mu)$ to $(\mu \lambda, \mu)$. Moreover the kernel on the fibre of some $\mu: Q_{0} \longrightarrow k^{d}$ is $\operatorname{Hom}_{A}\left(Q_{0}, \operatorname{ker} \mu\right)$ which has constant dimension as $\mu$ varies over $\operatorname{Surj}_{A}\left(Q_{0}, \mathbf{d}\right)$ since $Q_{0}$ is projective and the isomorphism class of $k e r \mu$ as an $A / \mathfrak{r}$-module is constant. Now the lemma follows from Proposition 6.

Let comproj $\left(A, \underline{d}_{1}\right)_{\mathbf{d}}$ be the $G$-stable locally closed subset of $\operatorname{comproj}\left(A, \underline{d}_{1}\right)$ consisting of complexes with degree zero homology in $\bmod (A, \mathbf{d})$.

Lemma 17. Let $V_{3}=\left\{(\lambda, \mu) \in V_{2} \mid\right.$ rank $\lambda$ is maximal $\}$. Then $V_{3}$ is open in $V_{2}$. Moreover

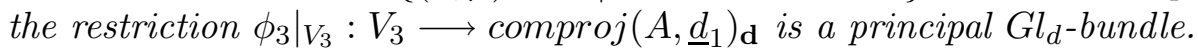

Proof. By the rank condition we know that $V_{3}$ is open in $V_{2}$. We prove the second statement. To simplify the notation we let $\rho$ denote $\left.\phi_{3}\right|_{V_{3}}$. Let $\lambda$ be in $\operatorname{comproj}\left(A, \underline{d}_{1}\right)_{\mathbf{d}}$. Let

$$
U_{\lambda}=\left\{\lambda^{\prime} \in \operatorname{comproj}\left(A, \underline{d}_{1}\right)_{\mathbf{d}} \mid i m \lambda^{\prime} \cap W=0 \text { and } \operatorname{ker} \lambda^{\prime} \cap V=0\right\},
$$

where $Q_{1}=V \oplus \operatorname{ker} \lambda$ and $Q_{0}=i m \lambda \oplus W$ as $A / \mathfrak{r}$-modules. Then $U_{\lambda}$ is an open neighbourhood of $\lambda$ in comproj $\left(A, \underline{d}_{1}\right)_{\mathbf{d}}$. For each $\left(\lambda^{\prime}, \mu\right) \in \rho^{-1}\left(U_{\lambda}\right)$ we can write

$$
\begin{gathered}
\lambda^{\prime}=\left(\begin{array}{cc}
\lambda_{1}^{\prime} & \lambda_{2}^{\prime} \\
\lambda_{3}^{\prime} & \lambda_{4}^{\prime}
\end{array}\right): V \oplus \operatorname{ker} \lambda \longrightarrow i m \lambda \oplus W \text { and } \\
\mu=\left(\mu_{1}, \mu_{2}\right): i m \lambda \oplus W \longrightarrow \mathrm{k}^{d} .
\end{gathered}
$$

So by the equation $\mu \lambda=0$ we have

$$
\begin{aligned}
& \mu_{1} \lambda_{1}^{\prime}+\mu_{2} \lambda_{3}^{\prime}=0, \\
& \mu_{1} \lambda_{2}^{\prime}+\mu_{2} \lambda_{4}^{\prime}=0 .
\end{aligned}
$$

Since $\operatorname{ker} \lambda^{\prime} \cap V=0$, we have an injection $\left(\begin{array}{c}\lambda_{1}^{\prime} \\ \lambda_{3}^{\prime}\end{array}\right): V \longrightarrow i m \lambda \oplus W$. Moreover $i m \lambda^{\prime} \cap W=0$, thus $\lambda_{1}^{\prime}$ is invertible and so $\mu_{1}=-\mu_{2} \lambda_{3}^{\prime}\left(\lambda_{1}^{\prime}\right)^{-1}$. Also $i m \lambda^{\prime}=i m\left(\begin{array}{c}\lambda_{1}^{\prime} \\ \lambda_{3}^{\prime}\end{array}\right)$. Hence $i m\left(\begin{array}{c}\lambda_{2}^{\prime} \\ \lambda_{4}^{\prime}\end{array}\right) \subseteq$ $i m\left(\begin{array}{l}\lambda_{1}^{\prime} \\ \lambda_{3}^{\prime}\end{array}\right)$. By the construction we know that $\mu_{2}$ may be identified with an element of $G l_{d}$. Therefore $\rho^{-1}\left(U_{\lambda}\right)=\left\{\left(\lambda^{\prime}, \mu\right) \mid \mu_{1}=-\mu_{2} \lambda_{3}^{\prime}\left(\lambda_{1}^{\prime}\right)^{-1}, \mu_{2} \in G l_{d}\right\}$. Hence there is an isomorphism from $\rho^{-1}\left(U_{\lambda}\right) \longrightarrow U_{\lambda}$ to the trivial bundle $U_{\lambda} \times G l_{d} \longrightarrow U_{\lambda}$ by sending $\left(\lambda^{\prime}, \mu\right)$ to $\left(\lambda^{\prime}, \mu_{2}\right)$. This shows that $\rho$ is a principal $G l_{d}$-bundle. 
We are finally able to prove Lemma 14 and therefore complete the proof of Theorem 2.

Proof of Lemma 14. We may identify $\operatorname{Surj}_{A}\left(Q_{0}, \mathbf{d}\right)$ with an open subset of $V_{1}$ by sending $\mu: Q_{0} \longrightarrow k^{d}$ to $(\pi(\mu), \mu)$. Note that since vector bundles are open morphisms Lemma 15 shows that $\operatorname{Fac}\left(Q_{0}, \mathbf{d}\right)$ is an open subset in $\bmod (A, \mathbf{d})$. Note that both $M$ and $N$ belong to $F a c\left(Q_{0}, \mathbf{d}\right)$ and so $\operatorname{Sing}(M, N)=\operatorname{Sing}\left(\overline{G l_{d} \cdot M} \cap \operatorname{Fac}\left(Q_{0}, \mathbf{d}\right), N\right)$.

Let $\psi$ denote the restriction of $\phi_{4}$ to $V_{3}$. Let

$$
\mathcal{Z}=(\pi \psi)^{-1}\left(\overline{G l_{d} \cdot M} \cap \operatorname{Fac}\left(Q_{0}, \mathbf{d}\right)\right) .
$$

By Lemma 15 and Lemma 16 we have $\operatorname{Sing}(M, N)=\operatorname{Sing}(\mathcal{Z}, Y)$ for any $Y \in \mathcal{Z}$ with $\pi \psi(Y) \simeq N$.

Like in the proof of Lemma 17 let $\rho$ denote the restriction of $\phi_{3}$ to $V_{3}$. Note that $(\lambda, \mu) \in V_{3}$ if and only if there is an exact sequence of $A$-modules

$$
Q_{0} \stackrel{\lambda}{\longrightarrow} Q_{1} \stackrel{\mu}{\longrightarrow} \pi(\mu) \longrightarrow 0
$$

So $(\pi \psi)^{-1}\left(G l_{d} \cdot M\right)=\rho^{-1}\left(G \cdot \pi_{1}\left(P_{M}\right)\right)$. We have

$$
\overline{\pi^{-1}\left(G l_{d} \cdot M\right)}=\pi^{-1}\left(\overline{G l_{d} \cdot M} \cap \operatorname{Fac}\left(Q_{0}, \mathbf{d}\right)\right) .
$$

For obviously $\overline{\pi^{-1}\left(G l_{d} \cdot M\right)} \subseteq \pi^{-1}\left(\overline{G l_{d} \cdot M} \cap F a c\left(Q_{0}, \mathbf{d}\right)\right)$. Now by Lemma $15 \pi^{-1}\left(\overline{G l_{d} \cdot M} \cap\right.$ $\left.\operatorname{Fac}\left(Q_{0}, \mathbf{d}\right)\right)$ is an irreducible closed subset of $\operatorname{Surj}_{A}\left(Q_{0}, \mathbf{d}\right)$ and so equality follows.

A similar argument using Lemma 16 gives

$$
\mathcal{Z}=\overline{(\pi \psi)^{-1}\left(G l_{d} \cdot M\right)} \text {. }
$$

Also

$$
\overline{\rho^{-1}\left(G \cdot \pi_{1}\left(P_{M}\right)\right)}=\rho^{-1}\left(\overline{G \cdot \pi_{1}\left(P_{M}\right)} \cap \operatorname{comproj}\left(A, \underline{d}_{1}\right)_{\mathbf{d}}\right)
$$

by Lemma 17. Thus

$$
\mathcal{Z}=\rho^{-1}\left(\overline{G \cdot \pi_{1}\left(P_{M}\right)} \cap \operatorname{comproj}\left(A, \underline{d}_{1}\right)_{\mathbf{d}}\right) .
$$

By Lemma 17 we have $\operatorname{Sing}\left(\pi_{1}\left(P_{M}\right), \pi_{1}\left(P_{N}\right)\right)=\operatorname{Sing}(\mathcal{Z}, Y)$ for any $Y \in \mathcal{Z}$ with $\rho(Y) \simeq$ $\pi_{1}\left(P_{N}\right)$. Moreover $\pi \psi(Y) \simeq H_{0}(\rho(Y))$ for any $Y \in \mathcal{Z}$. Hence $\operatorname{Sing}(M, N)=\operatorname{Sing}\left(\pi_{1}\left(P_{M}\right)\right.$, $\left.\pi_{1}\left(P_{N}\right)\right)$.

\section{Proof of Theorem 3}

As in the statement of the theorem, let $A$ be a hereditary $k$-algebra and let $M, N \in D^{b}(A)$ with homology of equal dimension. Assume that $M \leq_{\text {deg }} N$. Then there exist a complex $Z$ and a triangle $N \longrightarrow M \oplus Z \longrightarrow Z \longrightarrow N[1]$ in the derived category. We have a corresponding long exact sequence

$$
\ldots \longrightarrow H_{i}(N) \longrightarrow H_{i}(M) \oplus H_{i}(Z) \longrightarrow H_{i}(Z) \longrightarrow H_{i-1}(N) \longrightarrow \ldots
$$

in homology. Since $M$ and $N$ have homology of equal dimension and since $N$ is right bounded this long exact sequence splits into short exact sequences

$$
0 \longrightarrow H_{i}(N) \longrightarrow H_{i}(M) \oplus H_{i}(Z) \longrightarrow H_{i}(Z) \longrightarrow 0 .
$$

Hence $H_{*}(M) \leq_{\text {deg }} H_{*}(N)$. Conversely, if $H_{*}(M) \leq_{\text {deg }} H_{*}(N)$ there exists for each $i \in \mathbb{Z}$ a module $Z_{i}$ and a short exact sequence

$$
0 \longrightarrow H_{i}(N) \longrightarrow H_{i}(M) \oplus Z_{i} \longrightarrow Z_{i} \longrightarrow 0
$$

Let $Z=\bigoplus_{i \in \mathbb{Z}} Z_{i}$ be the corresponding graded module. If we view $H_{*}(N), H_{*}(M)$ and $Z$ as complexes with zero differentials we get a short exact sequence of complexes

$$
0 \longrightarrow H_{*}(N) \longrightarrow H_{*}(M) \oplus Z \longrightarrow Z \longrightarrow 0
$$

inducing an exact triangle $H_{*}(N) \longrightarrow H_{*}(M) \oplus Z \longrightarrow Z \longrightarrow H_{*}(N)$ [1] in the derived category. Now since $A$ is hereditary we have $M \simeq H_{*}(M)$ and $N \simeq H_{*}(N)$ and so $M \leq_{\text {deg }} N$. This completes the first part of the theorem.

Now suppose that $M, N \in \operatorname{comproj}(A, \underline{d})$ and $M \leq_{\text {deg }} N$, where $\underline{d}$ is bounded with $d^{i}=0$ for $i>m+1$ or $i<p$. We denote the projective module at degree $i$ by $Q_{i}$. Denote by $K_{i}$ the 
kernel of $\partial_{i}^{N}: Q_{i} \longrightarrow Q_{i-1}$. Since $A$ is hereditary, we have a decomposition $Q_{i}=K_{i} \oplus W_{i}$ as a direct sum of projective modules.

Without loss of generality we may assume that $p=0$. The proof is by induction on $m$. If $m=0$ then $\operatorname{Sing}(M, N)=\operatorname{Sing}\left(H_{0}(M), H_{0}(N)\right)$ by Lemma 14 . Now since $A$ is hereditary $H_{1}(M) \simeq H_{1}(N)$ in this case and so $H_{1}(N)$ is a smooth point in the orbit closure of $H_{1}(M)$. Hence $\operatorname{Sing}\left(H_{*}(M), H_{*}(N)\right)=\operatorname{Sing}(M, N)$.

We now do the induction step. Let $V$ be an $A / \mathfrak{r}$-module complement to $i m \partial_{m}^{N}$ in $Q_{m-1}$. Let $U$ be the subset of $\operatorname{comproj}(A, \underline{d})$ consisting of complexes $X$ with $\operatorname{ker}_{i}^{X} \cap W_{i}=0$ for $i=m-1, m, m+1, i m \partial_{m}^{X} \cap V=0$ and $H_{s}(X)$ and $H_{s}(M)$ isomorphic as $A / \mathfrak{r}$-modules, for all $s \in \mathbb{Z}$. For $X$ in $U$, we decompose $\partial_{m+1}^{X}$ and $\partial_{m}^{X}$ as

$\partial_{m+1}^{X}=\left(\begin{array}{ll}a & b \\ c & d\end{array}\right): K_{m+1} \oplus W_{m+1} \longrightarrow K_{m} \oplus W_{m}$ and $\partial_{m}^{X}=\left(\begin{array}{ll}e & f \\ g & h\end{array}\right): K_{m} \oplus W_{m} \longrightarrow i m \partial_{m}^{N} \oplus V$.

By the definition of $U$, we know that $b$ is injective and $f$ is bijective. So by the rank condition on $U$ we have $i m a \subseteq i m b$ and $i m e \subseteq i m f$. Also $c=-f^{-1} e a$ and $d=-f^{-1} e b$. Let $W \subseteq \operatorname{Hom}_{A}\left(K_{m+1} \oplus W_{m+1}, K_{m}\right)$ be the subvariety of maps $(a, b)$ with $i m a \subseteq i m b$ and $b$ injective. Then the rank of $b$ is equal to the rank of $\partial_{m+1}^{M}$. We have an isomorphism of varieties

$$
\psi_{1}: U \longrightarrow W \times \pi_{m}(U)
$$

given by sending $X$ to $\left((a, b), \pi_{m}(X)\right)$. That $\left(\begin{array}{cc}a & b \\ -f^{-1} e a & -f^{-1} e b\end{array}\right)$ is an $A$-homomorphism for any $(a, b) \in W$ follows from the condition $\operatorname{ker}_{m-1}^{X} \cap W_{m-1}=0$.

Let $\operatorname{Inj}_{A}\left(W_{m+1}, K_{m}\right)$ denote the injective $A$-homomorphisms from $W_{m+1}$ to $K_{m}$.

Lemma 18. Let $\psi_{2}: W \times \pi_{m}(U) \longrightarrow \operatorname{Inj}_{A}\left(W_{m+1}, K_{m}\right) \times \pi_{m}(U)$ denote the map given by $\psi_{2}\left((a, b), \pi_{m}(X)\right)=\left(b, \pi_{m}(X)\right)$. Then $\psi_{2}$ is a vector bundle.

Proof. It suffices to show that the map $\psi: W \longrightarrow \operatorname{Inj}_{A}\left(W_{m+1}, K_{m}\right)$ is a vector bundle. For $b \in \operatorname{Inj}_{A}\left(W_{m+1}, K_{m}\right)$, we define an open neighbourhood of $b$ by $U_{b}=\left\{b^{\prime} \mid i m b^{\prime} \cap P_{b}=0\right\}$, where $P_{b}$ is an $A / \mathfrak{r}$-module complement to $i m b$ in $K_{m}$. Let $b^{\prime} \in U_{b}$, we write

$$
b^{\prime}=\left(\begin{array}{l}
b_{1}^{\prime} \\
b_{2}^{\prime}
\end{array}\right): W_{m+1} \longrightarrow i m b \oplus P_{b} .
$$

By the definition of $U_{b}$, we know that $b_{1}^{\prime}$ is bijective.

For $a \in \operatorname{Hom}_{A}\left(K_{m+1}, K_{m}\right)$ we have $i m a \subseteq i m b^{\prime}$ if and only if $b^{\prime}\left(b_{1}^{\prime-1}, 0\right) a=a$. Let $\mu: U_{b} \times \operatorname{Hom}_{A}\left(K_{m+1}, K_{m}\right) \longrightarrow U_{b} \times \operatorname{Hom}_{k}\left(K_{m+1}, K_{m}\right)$ be defined by

$$
\mu\left(b^{\prime}, a\right)=\left(b^{\prime},\left(b^{\prime}\left(b_{1}^{\prime-1}, 0\right)-1\right) a\right) .
$$

Then $\mu$ is a morphism of vector bundles and $\psi^{-1}\left(U_{b}\right)=k e r \mu$. Now the fibre $\psi^{-1}\left(b^{\prime}\right)$ is isomorphic to $\operatorname{Hom}_{A}\left(K_{m+1}, i m b^{\prime}\right)$, which is constant since $i m b^{\prime}$ is constant as an $A / \mathfrak{r}$ module and $K_{m+1}$ is a projective $A$-module. Therefore $\psi$ is a vector bundle.

Now since $A$ is hereditary $\pi_{m}(\overline{G \cdot M} \cap U)=\overline{G \cdot \pi_{m}(M)} \cap \pi_{m}(U)$. For if $Y \in \overline{G \cdot \pi_{m}(M)} \cap$ $\pi_{m}(U)$, then $H_{i}\left(\pi_{m}(M)\right) \leq_{\text {deg }} H_{i}(Y)$ for $i \leq m$, and in particular $k e r \partial_{m}^{M} \cong k e r \partial_{m}^{Y}$. Then there exists $Y^{\prime} \in U$ with $\pi_{m}\left(Y^{\prime}\right)=Y$ and $H_{m}\left(Y^{\prime}\right) \simeq H_{m}(M)$, thus $M \leq_{\text {deg }} Y^{\prime}$. This shows $\pi_{m}(\overline{G \cdot M} \cap U) \supseteq \overline{G \cdot \pi_{m}(M)} \cap \pi_{m}(U)$. Now $\pi_{m}^{-1}\left(\overline{G \cdot \pi_{m}(M)}\right) \supseteq \pi_{m}^{-1}\left(G \cdot \pi_{m}(M)\right) \supseteq G \cdot M$ and so $\pi_{m}(\overline{G \cdot M} \cap U) \subseteq \overline{G \cdot \pi_{m}(M)} \cap \pi_{m}(U)$.

Now $H_{m}(X) \simeq K_{m} / i m b$ for any $X \in U$, where the isomorphism is induced by the composition $k e r \partial_{m}^{X} \subseteq K_{m} \oplus W_{m} \longrightarrow K_{m}$, which is an isomorphism by assumption.

Consequently,

$\psi_{2} \psi_{1}(\overline{G \cdot M} \cap U)=\left\{b \in \operatorname{Inj}_{A}\left(W_{m+1}, K_{m}\right) \mid H_{m}(M) \leq_{d e g} K_{m} / i m b\right\} \times\left(\pi_{m}(U) \cap \overline{G \cdot \pi_{m}(M)}\right)$

$\operatorname{Thus} \operatorname{Sing}(M, N)=\operatorname{Sing}\left(\psi_{2} \psi_{1}(\overline{G \cdot M} \cap U), \psi_{2} \psi_{1}(N)\right)$, since $\psi_{2}^{-1} \psi_{2} \psi_{1}(\overline{G \cdot M} \cap U)=$ $\psi_{1}(\overline{G \cdot M} \cap U)$ and $\operatorname{Sing}(M, N)=\operatorname{Sing}\left(\overline{G l_{d} \cdot H_{m}(M)} \times \overline{G \cdot \pi_{m}(M)},\left(H_{m}(N), \pi_{m}(N)\right)\right)$, by Lemma 14. 
Since $A$ is hereditary we have $H_{m+1}(M) \simeq H_{m+1}(N)$ and so $H_{m+1}(N)$ is a smooth point in the orbit closure of $H_{m+1}(M)$. Similarly $H_{m}\left(\pi_{m}(N)\right)$ is a smooth point in the orbit closure of $H_{m}\left(\pi_{m}(M)\right)$. Thus by induction $\operatorname{Sing}(M, N)=\operatorname{Sing}\left(\bigoplus_{i=0}^{m+1} H_{i}(M), \bigoplus_{i=0}^{m+1} H_{i}(N)\right)$.

\section{REFERENCES}

[1] Victor Bekkert and Yuri Drozd, Tame-wild dichotomy for derived categories, math.RT/0310352v1.

[2] Grzegorz Bobiński and Grzegorz Zwara, Normality of orbit closures for Dynkin quivers of type $\mathbb{A}_{n}$, Manuscripta Mathematica 105 (2001), no. 1, 103-109.

[3] Grzegorz Bobiński and Grzegorz Zwara, Schubert varieties and representations of Dynkin quivers, Colloquium Mathematicum 94 (2002), no. 2, 285-309.

[4] Klaus Bongartz, A geometric version of the Morita equivalence, Journal of Algebra 139 (1991) 159-171.

[5] Klaus Bongartz, Minimal singularities for representations of Dynkin quivers Commentarii Mathematici Helvetici 69 (1994), no. 4, 575-611.

[6] William Crawley-Boevey and Jan Schrer, Irreducible components of varieties of modules, Journal für die Reine und Angewandte Mathematik 553 (2002), 201-220.

[7] Wim Hesselink, Singularities in the nilpotent scheme of a classical group, Transactions of the American Mathematical Society 222 (1976), 1-32.

[8] Birge Huisgen-Zimmermann and Manuel Saorín Geometry of chain complexes and outer automorphisms under derived equivalence, Transactions of the American Mathematical Society 353 (2001), no. 12, 47574777

[9] Bernt Tore Jensen, Xiuping Su and Alexander Zimmermann, Degenerations for derived categories, Journal of Pure and Applied Algebra 198 (2005), no. 1-3, 281-295.

[10] Joseph Le Potier, Lectures on vector bundles, Translated by A. Maciocia. Cambridge Studies in Advanced Mathematics, 54. Cambridge University Press, Cambridge, 1997. viii+251 pp. ISBN: 0-521-48182-1

[11] Nicola J. Richmond, A stratification for varieties of modules, The Bulletin of the London Mathematical Society 33 (2001), no. 5, 565-577.

[12] Igor R. Shafarevich, Basic algebraic geometry. 2. Schemes and complex manifolds, Second edition, Translated from the 1988 Russian edition by Miles Reid. Springer-Verlag, Berlin, 1994. xiv+269 pp. ISBN: 3-540-57554-5

[13] Andrzej Skowroński and Grzegorz Zwara, Derived equivalences of selfinjective algebras preserve singularities, Manuscripta Mathematica 112 (2003), no. 2, 221-230.

[14] Grzegorz Zwara, Smooth morphisms of module schemes, Proceedings of the London Mathematical Society 84 (2002) 539-558.

Bernt Tore Jensen,

Department of Pure Mathematics,

UNIVERSITY OF LEEDS,

LEEDS LS2 9JT,

THE UNITED KINGDOM

E-mail address: bjensen@maths.leeds.ac.uk

XIUPing Su

UNIVERSité DE PicARdie,

LAMFA UMR 6140 DU CNRS,

(FACUlté De MathÉmatiques)

33 Rue St LeU,

F-80039 Amiens CEDEX 1,

FRANCE

E-mail address: xiuping.su@u-picardie.fr 